\title{
Insights into the Role of PAX-3 in the Development of Melanocytes and Melanoma
}

\author{
Jessica Diann Hathaway and Azizul Haque*
}

\author{
Department of Microbiology and Immunology, Charles Darby Children's Research Institute, and Hollings Cancer \\ Center, Medical University of South Carolina, 173 Ashley Avenue, Charleston, SC 29425, USA
}

\begin{abstract}
Melanoma is the deadliest form of skin cancer in the United States with an increasing prevalence. However, the development of melanoma from a melanocyte precursor is still poorly defined. Understanding the molecules responsible for melanoma progression may lead to improved targeted therapy. One potential molecule is the paired box-3 (PAX-3) protein, which has been implicated in the development of melanocytes and malignant melanoma. In melanoma, the expression of PAX-3 is believed to be differentially regulated, and has been linked with malignancies and staging of the disease. The loss of PAX-3 regulation has also been associated with the loss of transforming growth factor-beta (TGF- $\beta$ ) activity, but its effect on PAX-3 in differentiated melanocytes as well as metastatic melanoma remains unclear. Understanding PAX-3 regulation could potentially shift melanoma to a less aggressive and less metastatic disease. This review summarizes our current knowledge on PAX-3 during melanocyte development, its regulation, and its implications in the development of novel chemo-immunotherapeutics against metastatic melanoma.
\end{abstract}

Keywords: PAX-3, melanocyte, melanoma, SOX10, MITF, UVR, TGF- $\beta$.

\section{INTRODUCTION}

Melanoma is an aggressive skin cancer that has an occurrence rate of approximately 1 in every 50 Americans [1]. It is the $6^{\text {th }}$ most common cancer with a lifetime risk of $2.04 \%$ for men and $1.38 \%$ for women $[2,3]$. According to the American Cancer Society, in 2009 the number of new cases of melanoma rose to 68,720 with 8,650 new deaths attributed to the disease [4]. There are many known factors that contribute to the formation of melanoma. One major factor influencing the increase in melanoma cases is increased exposure to ultra-violet radiation (UVR); exposure resulting in severe sunburns leads to an increased incidence of melanoma [3]. Other risk factors include skin type, hair/eye color, the presence of dysplastic nevi and/or increased nevi, and a family history of melanoma [3]. Also, genetic mutations in BRAF, CDKN2A, and CDK4 have all been attributed to melanoma development. BRAF mutations have been found in $70 \%$ of all melanomas and greater than $90 \%$ of these mutations carry a single missense mutation [5, 6]. CDKN2A is a gene involved in melanoma pathogenesis and is a germline mutation found in younger patients [7]. CDK4 is involved in cell-cycle arrest and has been identified in $10 \%$ of observed melanomas [8]. These mutations are mostly detected in non-Chronic Sun-induced Damage melanomas, while Chronic Sun-induced Damage melanomas are more commonly observed.

When melanoma arises, early diagnosis is crucial to survival. With early diagnosis, more than $80 \%$ of cases can be treated successfully with surgery [9]. Surgery includes

*Address correspondence to this author at the Department of Microbiology and Immunology, Medical University of South Carolina, 173 Ashley Avenue, BSB-201, Charleston, SC 29425, USA; Tel: 843-792-9466; Fax: 843-792-2464; E-mail: haque@musc.edu excision of the tumor and surrounding tissue; lymph nodes near the tumor may also be removed if the cancer has metastasized. Other treatments for melanoma include radiation, used particularly in cases of metastasis, and chemotherapy, given when the cancer is highly aggressive and metastatic [4]. Side effects from these treatments include fatigue, malaise, and an increased susceptibility to non-melanoma cancers [10]. Also, these therapies are severely toxic to the patients, suggesting a need for improved and less toxic treatment options that specifically target melanoma tumors. Immunotherapy, such as IL-2 administration and adoptive T cell transfer, looks promising in filling this gap in treating metastatic melanoma [11]. Whole cell vaccines using melanoma antigens have also been studied in many forms with varied success [12-14]. But problems may occur with these Ags due to spontaneous cysteinylation of Ags, and disruption of HLA class II presentation [15-17]. Adoptive T cell transfer consists of an ex vivo expansion of autologous tumor reactive lymphocytes followed by re-infusion of these cells into the patient [18]. Adoptive T cell transfer has been successful in treating stage II melanoma, but not late stage melanoma [19]. Immunostimulating drug therapies have provided recent advances in targeting malignant melanoma. Ipilimumab is a human monoclonal antibody (IgG1) that targets CTLA-4 to promote antitumor immunity, and has shown improved outcome in metastatic melanoma patients [20]. Even with all of the current immunotherapy treatments, other potential targets that can be used to fight and treat late stage melanoma remain unknown.

One potential target may be the paired box-3 (PAX-3) protein, which is a member of the paired box family and an important regulator in melanocyte development. PAX-3 is a key transcription factor that plays a vital role in embryogenesis, but is also implicated in tumorigenesis [21]. It is expressed in early development, but inhibited in adult 
melanocytes. PAX-3 has been recognized as a key component in melanocyte development, yet its role in adult melanocytes and in melanoma remains unclear. In this review, we will discuss the nature of PAX-3 during the development and differentiation of melanocytes. We will also focus on the role of major regulators of PAX-3 in different stages of melanoma and its potential as a target for future therapies.

\section{MELANOCYTE DEVELOPMENT}

Melanocytes are derived from the neural crest during embryonic development and act as specialized pigment producing cells that are also the progenitor cells of melanoma $[22,23]$. During embryogenesis, melanocytic precursor cells emerge in the neural crest. From there, they migrate to populate various sites including the epidermis, hair follicles, the uveal tract of the eye, and the cochlea [6]. Wnt signaling is responsible for the promotion of neural crest cells into pigment cells and thus decides the fate of the unpigmented precursor melanoblast to mature into a melanocyte [24]. Melanocytes reside in the dermal layer of the skin and produce secretory vesicles called melanosomes, where concentrated and synthesized melanins are contained [25]. Melanins are then exported to the epidermal layer and surround the existing keratinocytes to form a protective layer [26]. The migration of melanin is responsible for the color and pigmentation of the skin. However, with excess UVR exposure, keratinocytes respond by signaling to nearby melanocytes, thus driving a cascade of signals toward differentiation [27]. PAX-3 plays an important role in this signaling cascade ultimately leading to the differentiation of melanocytes [28, 29]. However, specific pathways involved and the direct role of PAX-3 on the signaling of keratinocytes and melanocytes remains unknown.

\section{PAX-3 AND MELANOCYTOGENESIS}

PAX-3 is a crucial factor in the regulation of melanocytes during embryonic development [21, 30]. PAX-3 contains four domains: the paired domain, the homeodomain, the octapeptide, and the transactivation domain. The paired domain contains two helix-turn-helix (HTH) sub-domains named PAI and RED. The paired domain helps to facilitate protein-protein interactions and DNA binding [31]. The homeodomain, which contains three HTH motifs, may function to regulate the DNA binding ability of the paired domain [29, 32]. The octapeptide is comprised of eight amino acids, where it functions as a protein interaction epitope, linking the paired domain with the homeodomain [29]. The transactivation domain is located at the carboxylterminal end and acts as a mediator of PAX-3-DNA interactions. Neural crest cells are the first to express Pax-3 (italicized for gene expression), and expression continues when melanoblasts develop and migrate from the neural crest to hair follicles [33]. Also, when melanoblasts migrate from the neural crest to the epidermal/dermal border, they mature into melanocytes, having Pax-3, Sox10, Mitf, Dct, $T y r$, and TRP-1 gene expression, which may also play roles in melanocyte development $[34,35]$. PAX-3 is also important in neural crest specification, and later in the expansion of committed melanoblasts formed early in development $[36,37]$. Mutations in Pax-3 result in a disease called Waardenburg syndrome that includes abnormalities of the nervous system, eye, nose, as well as pigmentation defects affecting the skin, hair, and otic pigment cells [38, 39]. Many mutations within Pax-3 can result in Waardenburg syndrome, but the most common mutation is in the paired domain [40].

Despite the effect of PAX-3 on neural crest specification, its main and best described role is in the regulation of melanocyte differentiation [29, 37]. PAX-3 acts as both a transcriptional activator and repressor, and functions with other cofactors enhancing activation and repression [40]. The protein Sry-like HMG box 10 (SOX10) helps to enhance PAX-3's transcriptional activity by direct interaction [41, 42]. SOX10 is a member of the Sox family of transcription factors, and it contains an HMG DNA binding domain that directly interacts with the PAI sub-domain of the paired domain in the PAX-3 protein $[32,42]$. SOX10 is expressed in melanoblasts and melanocytes and its main function is in regulation of neural crest and melanocyte differentiation. PAX-3 directly interacts with SOX10, forming a complex which then binds to the promoter of Microphthalmia transcription factor (MITF). MITF is essential for melanoblast survival during and immediately following migration and is considered the major regulator during melanocytogenesis [37]. MITF activation is observed when the melanoblasts migrate to the epidermis [43]. In the melanoblasts, MITF activates the transcription of Dopachrome Tautomerase (Dct), Tyrosinase (Tyr), and Tyrosine related protein-1 (TRP-1), which may aid in early melanoblast differentiation. Taken together, PAX-3, SOX10, and MITF determine the stages of melanocyte differentiation, and regulate the maintenance of melanoblasts and melanocyte stem cells [34].

Not only does PAX-3 activate and regulate multiple proteins but it also represses certain proteins during melanocytogenesis (Fig. 1). As previously stated, PAX-3 binds with

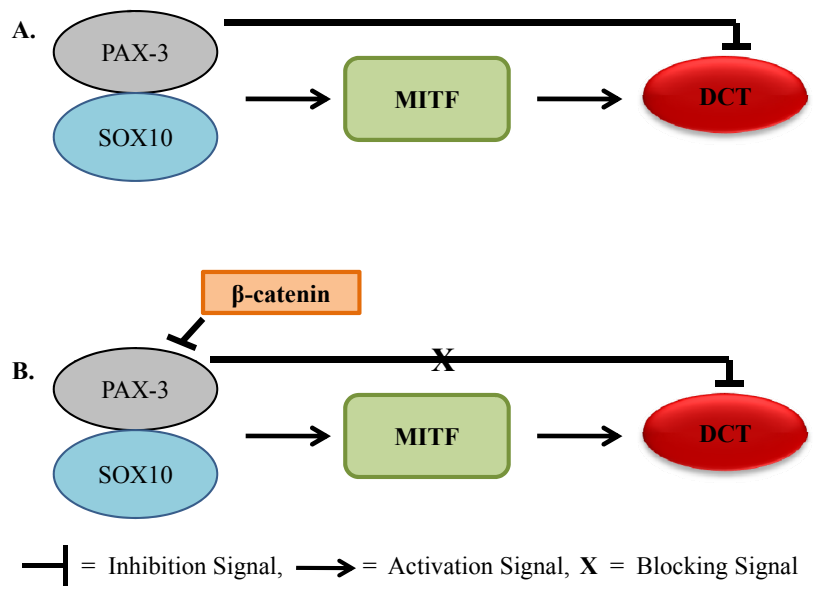

Fig. (1). Possible role of PAX-3 in melanocytogenesis. (A) During melanocytogenesis, PAX-3 forms a complex with SOX10 to activate MITF. MITF then binds to the Dct promoter to allow the melanocyte to differentiate. However, PAX-3 can also compete with MITF in binding to Det, and thus inhibiting it. (B) In the presence of $\beta$-catenin, the action of PAX-3 to block Dct transcription is lost. Det then contributes to melanocyte differentiation. 
SOX10 and together these activate MITF. MITF then activates Dct which encodes a melanogenic enzyme for melanoblasts [44]. However, PAX-3 can also block Dct expression, which then inhibits MITF to bind to the promoter of Dct [44]. This prevents terminal differentiation in the melanoblast, as PAX-3 and MITF share the same binding site on the Dct promoter and compete for binding [34]. Conversely, when beta-catenin is present, PAX-3 is displaced from the promoter of Dct [45]. This allows MITF to continuously activate Dct allowing melanocytes to fully differentiate. $\beta$ catenin is signaled by Wnt proteins whose function is in neural crest migration, proliferation, and differentiation [46]. Some of the ability of $\beta$-catenin to drive cells toward differentiation is through varying the function of PAX-3 and MITF [34, 47]. This may also explain PAX-3's role in melanoblast regulation and how PAX-3 is absent or significantly reduced in adult melanocytes.

\section{REGULATION \\ OF \\ PAX-3 \\ IN \\ ADULT}

PAX-3 expression is observed in early development, but not in adult melanocytes, as previously stated [21]. Regulation of PAX-3 is essential when melanocytes have differentiated into adult cells. One regulator of PAX-3 is TGF- $\beta$, a cytokine that controls numerous functions in multiple cells $[48,49]$. These functions include cellular behavior, differentiation, survival, migration, proliferation, and/or adhesion [50]. TGF- $\beta$ also protects homeostasis by acting as a tumor suppressor [51]. In melanoma cells, high levels of TGF- $\beta$ are secreted within the tumor microenvironment, but tumor cells fail to respond and growth inhibition is prevented [49]. While there are other regulators of PAX-3, TGF- $\beta$ has an important role in the maintenance of PAX-3 in adult melanocytes (Fig. 2). With limited UVR, keratinocytes residing in the epidermal layer of the skin secrete moderate

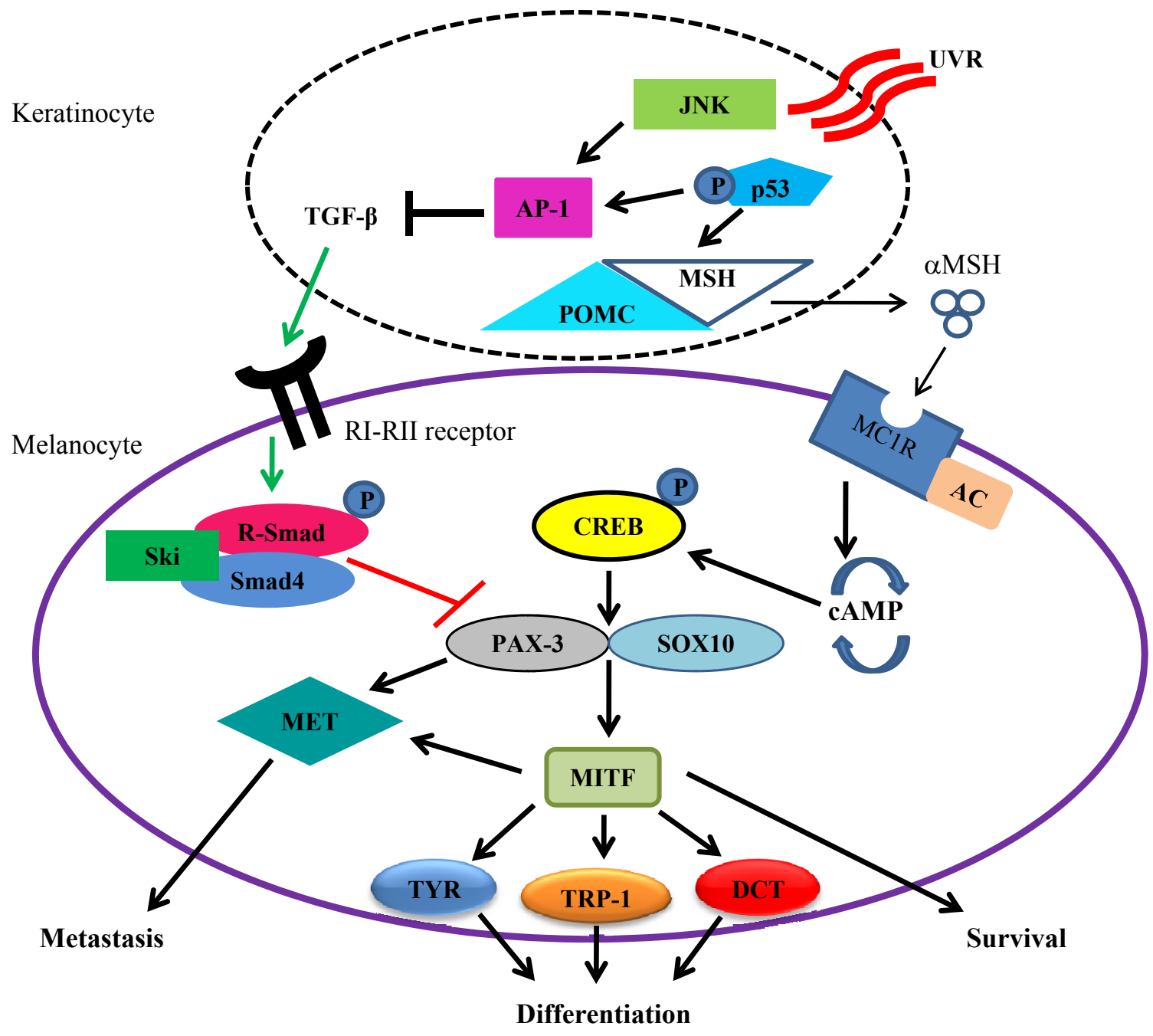

Fig. (2). Ultra-violet radiation and melanocyte differentiation. During normal melanocyte function, keratinocytes in the dermal layer of the skin produce TGF- $\beta$ that binds to the RI-RII receptor on the surface of melanocytes. This binding may signal phosphorylated R-Smad to form a complex with Smad4 and Ski, which inhibits the transcription of PAX-3. However, when UV radiation stimulates keratinocytes, both JNK and phosphorylated 53 respond by activating AP-1, which negatively regulates TGF- $\beta$. Phosphorylated p53 also induces POMC/MSH expression that allows $\alpha \mathrm{MSH}$ to be secreted for binding to the MC1R on the cell surface of melanocytes. MC1R, with the aid of adenylate cyclase, activates the cAMP pathway to phosphorylate CREB, which allows PAX-3 and SOX10 to bind together and induce MITF transcription. MITF then induces the transcription of the downstream targets TYR, TRP-1, and Dct, which are indications of melanocyte differentiation. Both MITF and PAX-3 can also activate MET, a protein that has been implicated in melanoma metastasis. 
levels of TGF- $\beta$, which signals through serine/threonine kinase receptor complexes (RI-RII) on the surface of melanocyte [52]. This binding at the cell membrane activates the Smad proteins to accumulate in the nucleus, which then controls targets of the TGF- $\beta /$ Smad complex $[26,53]$. Once TGF- $\beta$ is bound to the RI-RII receptor, R-Smad is phosphorylated and forms a complex with Smad4 and Ski [54]. Ski forms this complex in melanocytes and directly inhibits the transcription of PAX-3 [55]. A recent study also showed that treating melanocytes with varying concentrations of TGF- $\beta$ lead to the down-regulation of PAX-3 mRNA and protein expression [55]. This relationship between TGF- $\beta$ and PAX-3 will be further investigated in this review.

\section{PAX-3 AND MELANOMA}

Studies suggest that PAX-3 plays a critical role in melanocytogenesis [29, 34, 37, 47, 55]. However, it is not clear whether PAX-3 is involved in differentiated melanocytes and in melanoma. The expression of PAX-3 has been found in healthy melanocytes, in benign nevi, and in melanoma tumors [45, 56-58]. Because it is found in both benign and malignant lesions, the re-occurrence of PAX-3 expression may be involved in the transformation of normal melanocytes into malignant melanoma. PAX-3 expression in melanocytes of normal skin has been observed, which may result from TGF- $\beta$ activity loss in keratinocytes. Interestingly, PAX-3 has been used as a staging marker for melanoma and in the detection of circulating melanoma cells $[37,59]$. PAX-3 is also recognized as an immunogenic protein in melanoma $[60,61]$. It has also been found in primary tumors as well as in distinct stages of metastatic melanomas such as stages I and IV $[62,63]$. This indicates that the regulation of $\mathrm{PAX}-3$ could be a key factor in melanoma initiation, progression, and metastasis.

PAX-3 molecules could be involved in the progression of melanoma because of its role in cellular resistance to apoptosis. It has been shown that PAX-3 directly regulates the tumor suppressor protein PTEN, at least in myogenesis [64]. PTEN is involved in the progression through the G1 cell cycle check point by negatively regulating the PI3K/AKT signal transduction pathway [65]. This signaling pathway is responsible for regulating cell proliferation and resistance to apoptosis [29]. PAX-3 interacts with an alleged homeodomain binding motif within the PTEN promoter, which provides an opportunity for PAX-3 to modulate PTEN expression and ultimately allow for resistance to apoptosis [64]. This may be significant because PAX-3 expression in melanoma could induce resistance to apoptosis observed in highly malignant melanoma.

The loss of PAX-3 can also be linked with the loss of TGF- $\beta$ activity (Fig. 2). Levels of TGF- $\beta$ are reduced when keratinocytes are stimulated with UVR. Studies suggest that TGF- $\beta$ expression is also suppressed at both the transcriptional and protein level following UVR of the skin $[55,66]$. The repression of TGF- $\beta$ was caused by the UVR-induced expression of both Jun $\mathrm{N}$-terminal kinase (JNK) and the tumor suppressor p53. Collectively, JNK and p53 activate the transcription of activating protein 1 (AP-1) [67, 68]. This allows p53 to stimulate the production of pro-opiomelanocortin/melanocyte stimulating hormone (POMC/MSH) in keratinocytes $[27,69]$, which releases the $\alpha \mathrm{MSH}$ ligand that binds to the melanocortin-1 receptor (MC1R) on the melanocyte cell surface [69]. Once the $\alpha \mathrm{MSH}$ ligand binds to MC1R, a signal cascade that works with adenylate cyclase leads to the production of cyclic adenosine monophosphate (cAMP) [6]. As levels of cAMP reach a certain threshold, the phosphorylation of cAMP response element binding transcription factor (CREB) occurs via protein kinase A [69]. CREB stimulates both PAX-3 and SOX10 which together form a complex to activate MITF. MITF, the master regulator of melanocyte differentiation, plays multiple roles in melanoma. Loss of MITF results in cell cycle arrest and apoptosis, moderate levels lead to proliferation and survival, and increased expression results in differentiation [70, 71]. MITF and PAX-3 can also independently initiate the expression of receptor tyrosine kinase MET, which plays a role in cell migration and growth, and in cancer progression and metastasis [72].

TGF- $\beta$ plays an important role on PAX-3 in melanocytes, but loses its effect on melanoma cells that ultimately leads to melanocyte differentiation, metastasis, and survival [55]. However, the cause of the lost effect of TGF- $\beta$ on PAX-3 in melanoma is currently unknown. It has been suggested that when Ski localizes to the cytoplasm in melanocytes, effects of TGF- $\beta$ on the Smad complex are restricted and nuclear localization is prevented $[51,73]$. Further study may determine the role of TGF- $\beta$ in regulating PAX-3 in melanocytes and melanoma. The direct role that PAX-3 plays during intermediate stages of melanoma (i.e., stages II and III), must be identified to better understand the process of melanoma progression. Given PAX-3's expression in late stage metastatic melanoma but absence of intermediate stages, targeting PAX-3 could potentially shift the nature of melanoma tumors to a less aggressive, less metastatic form.

\section{CONCLUSIONS}

Melanoma is a deadly skin cancer that is becoming increasingly common. Treatments such as surgery, radiation, and chemotherapy have failed in curbing late stage metastatic disease. Immunotherapy has emerged to fill this void by providing a novel approach to treating metastatic melanoma [11]. Treatments such as IL-2 administration, whole cell vaccines, adoptive $\mathrm{T}$ cell transfer, and immunostimulating drug treatment have had some success in treating melanoma, but a standard of care remains elusive. The improved targeting of metastatic melanoma is needed for any of these techniques to be fully effective. The melanocyte protein PAX-3 is important in the regulation of melanocyte differentiation and is a potential target in melanoma. It has been implicated as a differentiation factor in melanoma, and has been increasingly used as a staging marker, with expression in stages I and IV. TGF- $\beta$ regulates PAX-3 through the activation of the Smad pathway. However, upon increased UVR exposure, TGF- $\beta$ functions are blocked, allowing PAX-3 molecules to activate downstream targets for melanocyte differentiation. In malignant melanoma, TGF- $\beta$ is still produced, but its effect is lost on PAX-3, allowing the protein to remain active. The targeting of PAX3 could potentially shift melanoma tumors to less aggressive and less metastatic form of the disease. PAX-3 siRNA could also block the differentiation, progression, and survival of 
the melanoma allowing a secondary treatment, such as immunotherapy or a combination of chemotherapy and immunotherapy to more successfully destroy the cancer. This review suggests that both in vitro and in vivo studies are needed to determine the specific activity of PAX-3 in the differentiation of melanocytes, and staging of malignant melanoma. Another direction of study could focus on the signaling pathways of TGF- $\beta$ and how its effect on PAX-3 is lost. Understanding the regulation of PAX-3 in the development of melanocytes and melanoma could provide an opportunity for drug targeting and advancement in the treatment of metastatic melanoma.

\section{ACKNOWLEDGEMENTS}

This work was supported by grants from the National Institutes of Health (CA129560 and CA129560-S1) to A. Haque. The authors also wish to acknowledge Bently Doonan, Drs. God and Radwan for critical reading of the manuscript.

\section{ABBREVIATIONS}

$$
\begin{aligned}
& \text { TGF- } \beta=\text { Transforming growth factor-beta } \\
& \text { UVR }=\text { Ultraviolet radiation } \\
& \text { CTLA-4 }=\text { Cytotoxic T lymphocyte-associated antigen } 4 \\
& \text { PAX-3 }=\text { Paired box } 3 \\
& \text { SOX10 }=\text { Sry-like HMG box } 10 \\
& \text { HTH }=\text { Helix-turn-helix } \\
& \text { MITF }=\text { Microphthalmia transcription factor } \\
& \text { Dct }=\text { Dopachrome tautomerase }
\end{aligned}
$$

\section{REFERENCES}

[1] Giblin AV, Thomas JM. Incidence, mortality and survival in cutaneous melanoma. J Plast Reconstr Aesthet Surg 2007; 60: 3240.

[2] Jemal A, Siegel R, Ward E, et al. Cancer statistics, CA. Cancer J Clin 2008; 58: 71-96.

[3] Jilaveanu LB, Aziz SA, Kluger HM. Chemotherapy and biologic therapies for melanoma: do they work? Clin Dermatol 2009; 27 : 614-25.

[4] Society AC. Cancer Facts \& Figures 2010. Available at: http://www.cancer.org/cancer/SkinCancer-Melanoma.

[5] Davies H, Bignell GR, Cox C et al. Mutations of the BRAF gene in human cancer. Nature 2002; 417: 949-54.

[6] Meyle KD, Guldberg P. Genetic risk factors for melanoma. Hum Genet 2009; 126: 499-510.

[7] Liu J, Suresh Kumar KG, Yu D et al. Oncogenic BRAF regulates beta-Trcp expression and NF-kappaB activity in human melanoma cells. Oncogene 2007; 26: 1954-8.

[8] Bennett DC. How to make a melanoma: what do we know of the primary clonal events? Pigment Cell Melanoma Res 2008; 21: 2738.

[9] Zhu S, Wurdak H, Wang Y et al. A genomic screen identifies TYRO3 as a MITF regulator in melanoma. Proc Natl Acad Sci USA 2009; 106: 17025-30.

[10] Kamposioras K, Pentheroudakis G, Pectasides D, Pavlidis N. Malignant melanoma of unknown primary site. To make the long story short. A systematic review of the literature. Crit Rev Oncol Hematol 2010;

[11] Mouawad R, Sebert M, Michels J et al. Treatment for metastatic malignant melanoma: old drugs and new strategies. Crit Rev Oncol Hematol 2010; 74: 27-39.
[12] Mitchell MS, Abrams J, Thompson JA, et al. Randomized trial of an allogeneic melanoma lysate vaccine with low-dose interferon Alfa-2b compared with high-dose interferon Alfa-2b for Resected stage III cutaneous melanoma. J Clin Oncol 2007; 25: 2078-85.

[13] Pan J, Jin P, Yan J, Kabelitz D. Anti-angiogenic active immunotherapy: a new approach to cancer treatment. Cancer Immunol Immunother 2008; 57: 1105-14.

[14] Yuan J, Ku GY, Gallardo HF, et al. Safety and immunogenicity of a human and mouse gp100 DNA vaccine in a phase I trial of patients with melanoma. Cancer Immun 2009; 9: 5.

[15] Haque MA, Hawes JW, Blum JS. Cysteinylation of MHC class II ligands: peptide endocytosis and reduction within APC influences T cell recognition. J Immunol 2001; 166: 4543-51.

[16] Norton DL, Haque A. Insights into the Role of GILT in HLA Class II Antigen Processing and Presentation by Melanoma. J Oncol 2009; 2009: 142959.

[17] Haque MA, Li P, Jackson SK, et al. Absence of gamma-interferoninducible lysosomal thiol reductase in melanomas disrupts $\mathrm{T}$ cell recognition of select immunodominant epitopes. J Exp Med 2002; 195: 1267-77.

[18] Jandus C, Speiser D, Romero P. Recent advances and hurdles in melanoma immunotherapy. Pigment Cell Melanoma Res 2009; 22: 711-23.

[19] Bendle GM, Haanen JB, Schumacher TN. Preclinical development of T cell receptor gene therapy. Curr Opin Immunol 2009; 21: 20914.

[20] Hodi FS, O'Day SJ, McDermott DF et al. Improved Survival with Ipilimumab in Patients with Metastatic Melanoma. N Engl J Med 2010; 8: 711-23.

[21] Lang D, Powell SK, Plummer RS, Young KP, Ruggeri BA. PAX genes: roles in development, pathophysiology, and cancer. Biochem Pharmacol 2007; 73: 1-14.

[22] Dahl C, Guldberg P. The genome and epigenome of malignant melanoma. APMIS 2007; 115: 1161-76.

[23] Uong A, Zon LI. Melanocytes in development and cancer. J Cell Physiol 2010; 222: 38-41.

[24] Dupin E, Glavieux C, Vaigot P, Le Douarin NM. Endothelin 3 induces the reversion of melanocytes to glia through a neural crestderived glial-melanocytic progenitor. Proc Natl Acad Sci USA 2000; 97: 7882-7.

[25] Lin JY, Fisher DE. Melanocyte biology and skin pigmentation. Nature 2007; 445: 843-50.

[26] Moustakas A, Heldin CH. Dynamic control of TGF-beta signaling and its links to the cytoskeleton. FEBS Lett 2008; 582: 2051-65.

[27] Moustakas A. TGF-beta targets PAX3 to control melanocyte differentiation. Dev Cell 2008; 15: 797-9.

[28] Blake JA, Thomas M, Thompson JA, White R, Ziman M. Perplexing Pax: from puzzle to paradigm. Dev Dyn 2008; 237: 2791-803.

[29] Kubic JD, Young KP, Plummer RS, Ludvik AE, Lang D. Pigmentation PAX-ways: the role of Pax3 in melanogenesis, melanocyte stem cell maintenance, and disease. Pigment Cell Melanoma Res 2008; 21: 627-45.

[30] Robson EJ, He SJ, Eccles MR. A PANorama of PAX genes in cancer and development. Nat Rev Cancer 2006; 6: 52-62.

[31] Birrane G, Soni A, Ladias JA. Structural basis for DNA recognition by the human PAX3 homeodomain. Biochemistry 2009; 48: 114855 .

[32] Cao Y, Wang C. The COOH-terminal transactivation domain plays a key role in regulating the in vitro and in vivo function of Pax3 homeodomain. J Biol Chem 2000; 275: 9854-62.

[33] Blake JA, Ziman MR. Pax3 transcripts in melanoblast development. Dev Growth Differ 2005; 47: 627-35.

[34] Lang D, Lu MM, Huang L, et al. Pax3 functions at a nodal point in melanocyte stem cell differentiation. Nature 2005; 433: 884-7.

[35] Wang Q, Fang WH, Krupinski J, et al. Pax genes in embryogenesis and oncogenesis. J Cell Mol Med 2008; 12: 2281-94.

[36] Hornyak TJ, Hayes DJ, Chiu LY, Ziff EB. Transcription factors in melanocyte development: distinct roles for Pax-3 and Mitf. Mech Dev 2001; 101: 47-59.

[37] Medic S, Ziman M. PAX3 across the spectrum: from melanoblast to melanoma. Crit Rev Biochem Mol Biol 2009; 44: 85-97.

[38] Waardenburg PJ. A new syndrome combining developmental anomalies of the eyelids, eyebrows and nose root with pigmentary defects of the iris and head hair and with congenital deafness. Am J Hum Genet 1951; 3: 195-253. 
[39] Wollnik B, Tukel T, Uyguner O, et al. Homozygous and heterozygous inheritance of PAX3 mutations causes different types of Waardenburg syndrome. Am J Med Genet A 2003; 122A: 42-5.

[40] Pingault V, Ente D, Dastot-Le Moal F, et al. Review and update of mutations causing Waardenburg syndrome. Hum Mutat 2010; 31: 391-406.

[41] Lang D, Epstein JA. Sox 10 and Pax3 physically interact to mediate activation of a conserved c-RET enhancer. Hum Mol Genet 2003; 12: 937-45.

[42] Mollaaghababa R, Pavan WJ. The importance of having your SOX on: role of SOX10 in the development of neural crest-derived melanocytes and glia. Oncogene 2003; 22: 3024-34.

[43] Palmieri G, Capone M, Ascierto ML, et al. Main roads to melanoma. J Transl Med 2009; 7: 86.

[44] Cheli Y, Ohanna M, Ballotti R, Bertolotto C. Fifteen-year quest for microphthalmia-associated transcription factor target genes. Pigment Cell Melanoma Res 2010; 23: 27-40.

[45] Medic S, Ziman M. PAX3 expression in normal skin melanocytes and melanocytic lesions (naevi and melanomas). PLoS One 2010; 5: e9977.

[46] Wu WB, Peng HC, Huang TF. Disintegrin causes proteolysis of beta-catenin and apoptosis of endothelial cells. Involvement of cellcell and cell-ECM interactions in regulating cell viability. Exp Cell Res 2003; 286: 115-27.

[47] Schepsky A, Bruser K, Gunnarsson GJ, et al. The microphthalmiaassociated transcription factor Mitf interacts with beta-catenin to determine target gene expression. Mol Cell Biol 2006; 26: 8914-27.

[48] Shirakata Y. Regulation of epidermal keratinocytes by growth factors. J Dermatol Sci 2010.

[49] Fuxe J, Vincent T, de Herreros AG. Transcriptional crosstalk between TGFbeta and stem cell pathways in tumor cell invasion: Role of EMT promoting Smad complexes. Cell Cycle 2010; 9:

[50] ten Dijke P, Hill CS. New insights into TGF-beta-Smad signalling. Trends Biochem Sci 2004; 29: 265-73.

[51] Chen D, Lin Q, Box N, et al. SKI knockdown inhibits human melanoma tumor growth in vivo. Pigment Cell Melanoma Res 2009; 22: 761-72.

[52] Zhao W, Gomez G, Yu SH, Ryan JJ, Schwartz LB. TGF-beta1 attenuates mediator release and de novo Kit expression by human skin mast cells through a Smad-dependent pathway. J Immunol 2008; 181: 7263-72.

[53] Siegel PM, Massague J. Cytostatic and apoptotic actions of TGFbeta in homeostasis and cancer. Nat Rev Cancer 2003; 3: 807-21.

[54] Stavroulaki M, Kardassis D, Chatzaki E, et al. Exposure of normal human melanocytes to a tumor promoting phorbol ester reverses growth suppression by transforming growth factor beta. J Cell Physiol 2008; 214: 363-70.

[55] Yang G, Li Y, Nishimura EK, et al. Inhibition of PAX3 by TGFbeta modulates melanocyte viability. Mol Cell 2008; 32: 554-63.

[56] Scholl FA, Kamarashev J, Murmann OV, et al. PAX3 is expressed in human melanomas and contributes to tumor cell survival. Cancer Res 2001; 61: 823-6.
[57] Muratovska A, Zhou C, He S, Goodyer P, Eccles MR. Paired-Box genes are frequently expressed in cancer and often required for cancer cell survival. Oncogene 2003; 22: 7989-97.

[58] Plummer RS, Shea CR, Nelson M, et al. PAX3 expression in primary melanomas and nevi. Mod Pathol 2008; 21: 525-30.

[59] Koyanagi K, Kuo C, Nakagawa T, et al. Multimarker quantitative real-time PCR detection of circulating melanoma cells in peripheral blood: relation to disease stage in melanoma patients. Clin Chem 2005; 51: 981-8.

[60] Rodeberg DA, Nuss RA, Elsawa SF, Erskine CL, Celis E. Generation of tumoricidal PAX3 peptide antigen specific cytotoxic T lymphocytes. Int J Cancer 2006; 119: 126-32.

[61] Himoudi N, Nabarro S, Yan M, et al. Development of anti-PAX3 immune responses; a target for cancer immunotherapy. Cancer Immunol Immunother 2007; 56: 1381-95.

[62] Ryu B, Kim DS, Deluca AM, Alani RM. Comprehensive expression profiling of tumor cell lines identifies molecular signatures of melanoma progression. PLoS One 2007; 2: e594.

[63] Hilari JM, Mangas C, Xi L, et al. Molecular staging of pathologically negative sentinel lymph nodes from melanoma patients using multimarker, quantitative real-time rt-PCR. Ann Surg Oncol 2009; 16: 177-85.

[64] Li HG, Wang Q, Li HM, et al. PAX3 and PAX3-FKHR promote rhabdomyosarcoma cell survival through downregulation of PTEN. Cancer Lett 2007; 253: 215-23.

[65] Chow LM, Baker SJ. PTEN function in normal and neoplastic growth. Cancer Lett 2006; 241: 184-96.

[66] Quan T, He T, Kang S, Voorhees JJ, Fisher GJ. Solar ultraviolet irradiation reduces collagen in photoaged human skin by blocking transforming growth factor-beta type II receptor/Smad signaling. Am J Pathol 2004; 165: 741-51.

[67] Cui R, Widlund HR, Feige E, et al. Central role of p53 in the suntan response and pathologic hyperpigmentation. Cell 2007; 128: 853-64.

[68] Gambichler T, Skrygan M, Tomi NS, et al. Significant downregulation of transforming growth factor-beta signal transducers in human skin following ultraviolet-A1 irradiation. $\mathrm{Br} \mathrm{J}$ Dermatol 2007; 156: 951-6.

[69] Sommers JA, Sharma S, Doherty KM, et al. p53 modulates RPAdependent and RPA-independent WRN helicase activity. Cancer Res 2005; 65: 1223-33.

[70] McGill GG, Horstmann M, Widlund HR, et al. Bcl2 regulation by the melanocyte master regulator Mitf modulates lineage survival and melanoma cell viability. Cell 2002; 109: 707-18.

[71] Carreira S, Goodall J, Denat L, et al. Mitf regulation of Dia1 controls melanoma proliferation and invasiveness. Genes Dev 2006; 20: 3426-39.

[72] Mascarenhas JB, Littlejohn EL, Wolsky RJ, et al. PAX3 and SOX10 activate MET receptor expression in melanoma. Pig Cell Melanoma Res 2010; 23: 225-37.

[73] Kokura K, Kim H, Shinagawa T, et al. The Ski-binding protein $\mathrm{C} 184 \mathrm{M}$ negatively regulates tumor growth factor-beta signaling by sequestering the Smad proteins in the cytoplasm. J Biol Chem 2003; 278: 20133-9.

Received: August 30, 2010

Revised: November 04, 2010

Accepted: November 06, 2010

(C) Hathaway and Haque; Licensee Bentham Open.

This is an open access article licensed under the terms of the Creative Commons Attribution Non-Commercial License (http://creativecommons.org/licenses/by$\mathrm{nc} / 3.0 /$ ), which permits unrestricted, non-commercial use, distribution and reproduction in any medium, provided the work is properly cited. 